\title{
Endoplasmic reticulum refilling and mitochondrial calcium extrusion promoted in neurons by NCX1 and NCX3 in ischemic preconditioning are determinant for neuroprotection
}

\author{
MJ Sisalli ${ }^{1}$, A Secondo ${ }^{1}$, A Esposito ${ }^{1}$, V Valsecchi ${ }^{1}$, C Savoia ${ }^{1}$, GF Di Renzo ${ }^{1}$, L Annunziato ${ }^{1}$ and A Scorziello*,1
}

Ischemic preconditioning (IPC), an important endogenous adaptive mechanism of the CNS, renders the brain more tolerant to lethal cerebral ischemia. The molecular mechanisms responsible for the induction and maintenance of ischemic tolerance in the brain are complex and still remain undefined. Considering the increased expression of the two sodium calcium exchanger (NCX) isoforms, NCX1 and NCX3, during cerebral ischemia and the relevance of nitric oxide (NO) in IPC modulation, we investigated whether the activation of the NO/PI3K/Akt pathway induced by IPC could regulate calcium homeostasis through changes in NCX1 and NCX3 expression and activity, thus contributing to ischemic tolerance. To this aim, we set up an in vitro model of IPC by exposing cortical neurons to a 30-min oxygen and glucose deprivation (OGD) followed by 3-h OGD plus reoxygenation. IPC was able to stimulate NCX activity, as revealed by Fura-2AM single-cell microfluorimetry. This effect was mediated by the NO/PI3K/ Akt pathway since it was blocked by the following: (a) the NOS inhibitors L-NAME and 7-Nitroindazole, (b) the IP3K/Akt inhibitors LY294002, wortmannin and the Akt-negative dominant, (c) the NCX1 and NCX3 siRNA. Intriguingly, this IPC-mediated upregulation of NCX1 and NCX3 activity may control calcium level within endoplasimc reticulum (ER) and mitochondria, respectively. In fact, IPC-induced NCX1 upregulation produced an increase in ER calcium refilling since this increase was prevented by siNCX1. Moreover, by increasing NCX3 activity, IPC reduced mitochondrial calcium concentration. Accordingly, the inhibition of NCX by CGP37157 reverted this effect, thus suggesting that IPC-induced NCX3-increased activity may improve mitochondrial function during OGD/reoxygenation. Collectively, these results indicate that IPC-induced neuroprotection may occur through the modulation of calcium homeostasis in ER and mitochondria through NO/PI3K/Akt-mediated NCX1 and NCX3 upregulation.

Cell Death and Differentiation (2014) 21, 1142-1149; doi:10.1038/cdd.2014.32; published online 14 March 2014

Ischemic preconditioning (IPC), an important endogenous adaptive mechanism of the brain, increases neuronal tolerance to lethal cerebral ischemia. The molecular mechanisms responsible for inducing and maintaining ischemic tolerance in the brain are complex and are not yet fully understood. Among the three isoforms of the $\mathrm{Na}^{+} / \mathrm{Ca}^{2+}$ exchanger, NCX1 and NCX3 represent two new possible molecular effectors involved in the neuroprotective mechanisms of IPC. ${ }^{1-3}$ Indeed, the increased expression of these two plasma membrane proteins, which have a fundamental role in regulating and maintaining cellular calcium and sodium homeostasis in the brain ${ }^{4,5}$ during IPC, has been associated with a decrease in the infarct volume following a more severe ischemic insult. ${ }^{1}$ However, the molecular mechanisms by which NCX1 and NCX3 upregulation lead to IPC-induced brain tolerance still remain unexplored.

In vitro experiments performed in cortical neurons exposed to oxygen and glucose deprivation (OGD) and subsequent reoxygenation have demonstrated that changes in $\mathrm{NCX}$ isoform expression during OGD are accompanied by increases in both NCX1 activity and endoplasimc reticulum (ER) $\mathrm{Ca}^{2+}$ refilling. ${ }^{6}$ Considering the increased expression of the two sodium calcium exchanger (NCX) isoforms, NCX1 and NCX3, during cerebral ischemia and the relevance of nitric oxide (NO) in IPC modulation, ${ }^{7,8}$ we investigated whether the activation of the NO/PI3K/Akt pathway induced by IPC could regulate calcium homeostasis through changes in NCX1 and NCX3 expression and activity, thus contributing to ischemic tolerance.

More recently, we have reported that among the three NCX isoforms, only NCX3 is expressed on the outer mitochondrial membrane, where it works mainly by extruding calcium from the matrix. ${ }^{9}$ In this regard, an even more compelling result is that NCX3 gene ablation induces not only the disappearance of the protein from the OMM but also the accumulation of mitochondrial calcium in cortical neurons. Interestingly, NCX3 expression decreases in cortical neurons during OGD, a finding that correlates with an increase in $\left[\mathrm{Ca}^{2+}\right]_{\mathrm{m}} \cdot{ }^{9}$

\footnotetext{
${ }^{1}$ Division of Pharmacology, Department of Neuroscience, Reproductive and Dentistry Sciences, School of Medicine, Federico II University of Naples - National Institute of Neuroscience, Naples, Italy

*Corresponding author: A Scorziello, Division of Pharmacology, Department of Neuroscience, Reproductive and Dentistry Sciences, School of Medicine, Federico II University of Naples - National Institute of Neuroscience, Via Sergio Pansini 5, 80131 Naples, Italy. Tel: +39 81 7463330; Fax: +39 81 7463323; E-mail: scorziel@ unina.it Abbreviations: IPC, ischemic preconditioning; NCX, sodium calcium exchanger; OGD, oxygen and glucose deprivation; L-NAME, L-NG-Nitroarginine methyl ester; 7NI, 7-Nitroindazole; WMN, wortmannin; OMM, outer mitochondrial membrane; ER, endoplasimc reticulum; Tg, Thapsigargin; CGP, 7-Chloro-5-(2-chlorophenyl)-1,5dihydro-4,1-benzothiazepin-2(3H)-one; EBSS, Earle's Balanced Salt Solution; FCS, fetal calf serum; HS, horse serum; NO, nitric oxide

Received 30.9.13; revised 15.1.14; accepted 04.2.14; Edited by N Bazan; published online 14.3.14
} 
Furthermore, preserving mitochondrial function is relevant for preconditioning-induced neuroprotection. In fact, preconditioning positively affects the integrity of mitochondrial oxidative phosphorylation after cerebral ischemia, ${ }^{10}$ prevents mitochondrial swelling, protects mitochondrial energy metabolism during cerebral ischemia by avoiding ATP consumption $^{11}$ and increases Mn-SOD expression and activity through the NO/Ras/ERK1-2 pathway. ${ }^{8}$

Although mitochondria are considered to be important mediators of endogenous neuroprotection, the mechanisms by which they might integrate cytoprotective signaling of preconditioning still remain to be fully elucidated. Thus, we investigated the role played by NCX1 and NCX3 in regulating $\mathrm{ER}$ and mitochondrial calcium homeostasis as a novel mechanism responsible for IPC-induced neuroprotection.

For this aim, cortical neurons were exposed to $30 \mathrm{~min}$ of OGD followed by 3-h OGD plus reoxygenation. The expression and activity of NCX1 and NCX3 were observed by means of western blot analysis, confocal microscopy and single cell microfluorimetry. The results showed that IPC-induced neuroprotection occurs through the modulation of calcium homeostasis in ER and mitochondria through NO/Aktmediated NCX1 and NCX3 upregulation.

\section{Results}

IPC induces upregulation of NCX1 and NCX3 expression and reverses OGD-induced downregulation. Exposing cortical neurons to $30 \mathrm{~min}$ of OGD, a condition that is comparable to a sublethal ischemic insult commonly defined as IPC, increased NCX1 and NCX3 protein expression $48 \mathrm{~h}$ later. When cortical neurons were exposed to IPC followed by OGD/reoxygenation, the significant increase in NCX1 and NCX3 expression was still present (Figures 1a and b). To understand whether IPC might affect NCX expression at the transcriptional level, NCX1 and NCX3 mRNA were detected using RT-PCR $48 \mathrm{~h}$ after IPC. The results showed that after IPC, NCX1 mRNA increased, whereas NCX3 mRNA remained unchanged (Figures $1 c$ and $d$ ).

NO mediates IPC-induced upregulation of NCX1 and NCX3 expression. As NCX1 and NCX3 constitute novel additional targets for the prosurvival action of the Akt/Protein Kinase B pathway, ${ }^{12}$ and as IPC induces NO production and Akt activation, ${ }^{13}$ the involvement of the NO/Akt pathway in the regulation of NCX expression was explored in cortical neurons during IPC. Interestingly, IPC caused an increase in the phosphorylated form of Akt, which was abolished after the treatment with nNOS inhibitors L-NAME $^{14}$ and 7-Nitroindazole $(7 \mathrm{NI})^{15}$ (Figure 1e, Supplementary Figure S1C).

To demonstrate that NO-induced Akt activation was responsible for the increased expression of NCX1 and NCX3 during IPC, further experiments were performed in cortical neurons first treated with L-NAME $(500 \mu \mathrm{M})$ and $7 \mathrm{NI}$ $(500 \mu \mathrm{M})$ to inhibit nNOS, or with LY294002 $(25 \mu \mathrm{M})$, wortmannin (WMN, $1 \mu \mathrm{M}$ ) and Akt-negative dominant (Akt D-) to inhibit the PI3K/Akt pathway, ${ }^{12}$ and then exposed to IPC. As reported in Figure 2 and Supplementary Figure S1, the inhibition of nNOS prevents IPC-induced NCX1 and NCX3 expression, whereas L-NAME prevented only IPC-induced NCX3 overexpression (Figures $2 a$ and b, Supplementary Figures S1A and B). Similarly, the block of the PI3/Akt pathway also counteracted the effects of IPC on NCX1 and NCX3 expression (Figures 2a and b; Supplementary Figures $\mathrm{S} 1 \mathrm{~A}$ and $\mathrm{B})$. This finding was supported by the transfection of the neurons with the Akt-negative dominant (Supplementary Figure S1D). Conversely, LY294002, but not L-NAME, also counteracted the effects of IPC on NCX1 and NCX3 mRNA expression (Figures $2 \mathrm{c}$ and d).

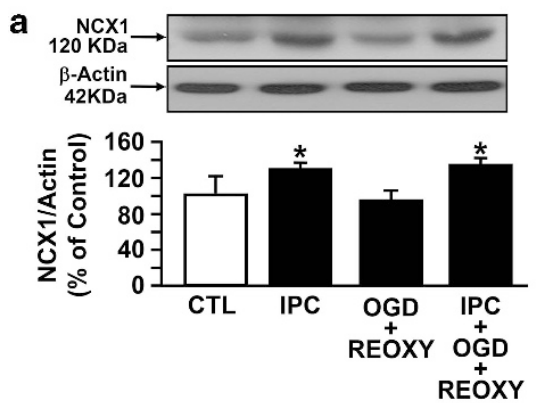

C

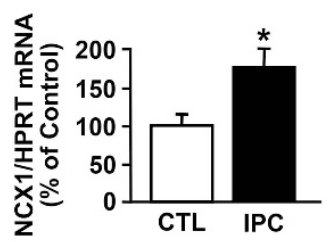

d

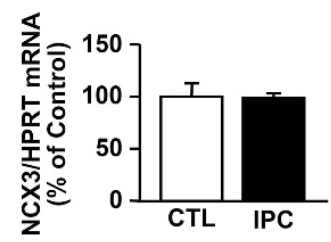

b
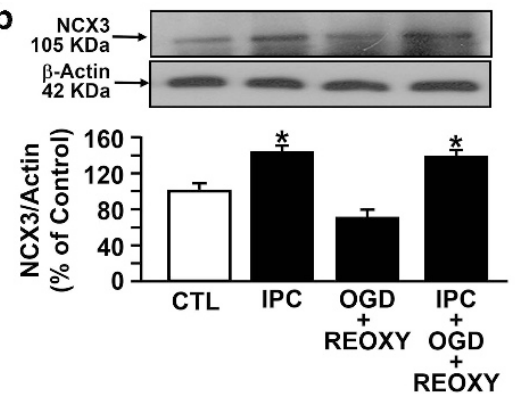

e

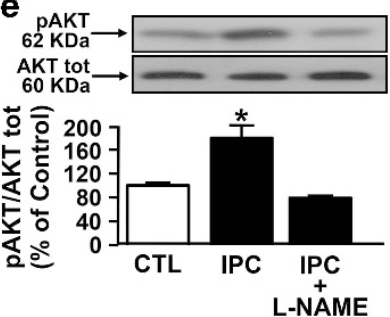

Figure 1 NCX1 e NCX3 and Akt expression after IPC in cortical neurons exposed to OGD/reoxygenation. Cortical neurons (12 DIV) were exposed to IPC (30-min OGD) followed by $48 \mathrm{~h}$ of normoxia and to $3 \mathrm{~h}$ of OGD followed by $24 \mathrm{~h}$ of reoxygenation. Protein and transcript expression were evaluated by western blotting and RT-PCR, respectively, at the end of reoxygenation. (a and $\mathbf{b})$ NCX1 and NCX3 expression. ${ }^{*} P<0.05$ versus $\mathrm{CTL}$ and OGD/REOXY. (c and $\mathbf{d}$ ) NCX1 and NCX3 transcripts. ${ }^{*} P<0.05$ versus CTL. (e) Akt activation in the presence of L-NAME. ${ }^{*} P<0.05$ versus $C T L$ and IPC + L-NAME 
NO-dependent NCX1 activation induced by IPC is responsible for ER calcium refilling in cortical neurons. To assess NCX activity during ischemic tolerance, parallel studies were carried out by using single cell Fura-2 AM microfluorimetry. IPC increased NCX activity in the reverse mode of operation 30 min after the sublethal insult, an effect that persisted for $48 \mathrm{~h}$ (Figure 3a). Conversely, NCX reverse mode of operation was impaired in cortical neurons exposed to OGD/reoxygenation but returned to the basal level in preconditioned neurons exposed to OGD/reoxygenation (Figure 3a). Interestingly, the reverse activity of NCX occurring in preconditioned neurons did not affect
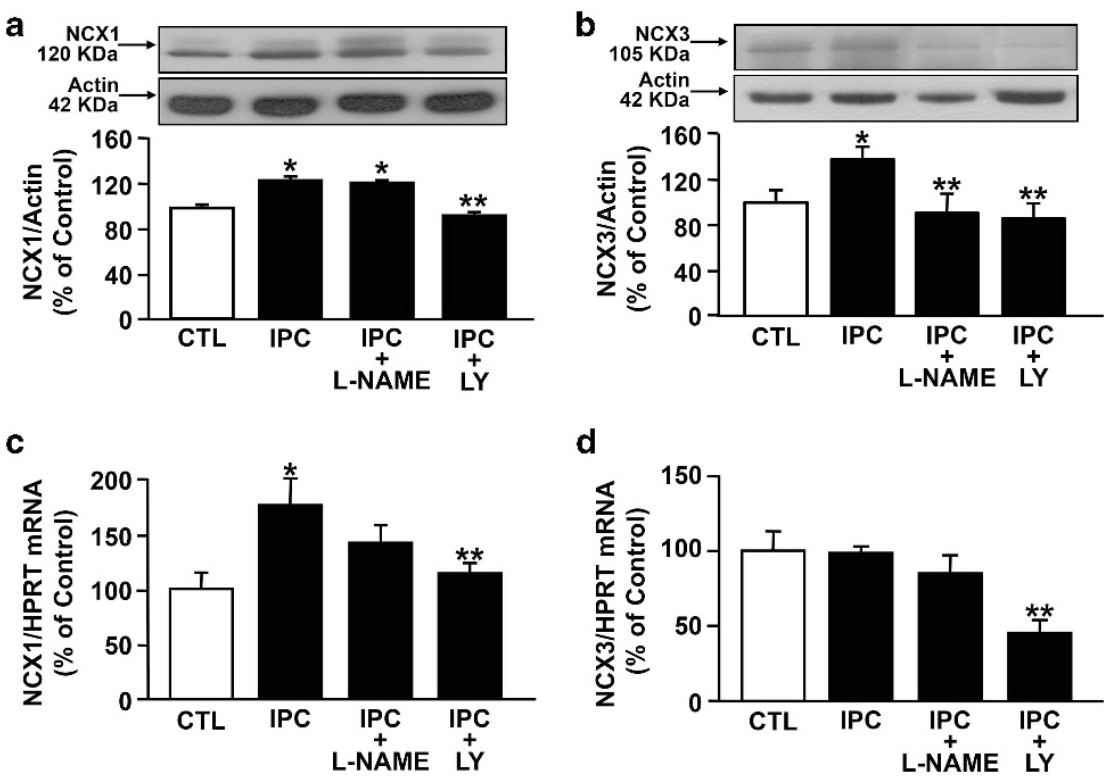

Figure 2 NO and Akt involvement in the regulation of NCX1 and NCX3 expression during IPC. Cortical neurons (12 DIV) were exposed to IPC (30 min OGD) with and without L-NAME and LY294002. Protein and transcript expression were evaluated by western blotting and RT-PCR, respectively, $48 \mathrm{~h}$ after the IPC. (a and b) IPC-induced NCX1 and NCX3 expression in the presence of L-NAME and LY294002, respectively. (c and d) IPC-induced NCX1 and NCX3 transcript expression in the presence of L-NAME and LY294002, respectively. ${ }^{*} P<0.05$ versus $C T L ;{ }^{* *} P<0.05$ versus IPC
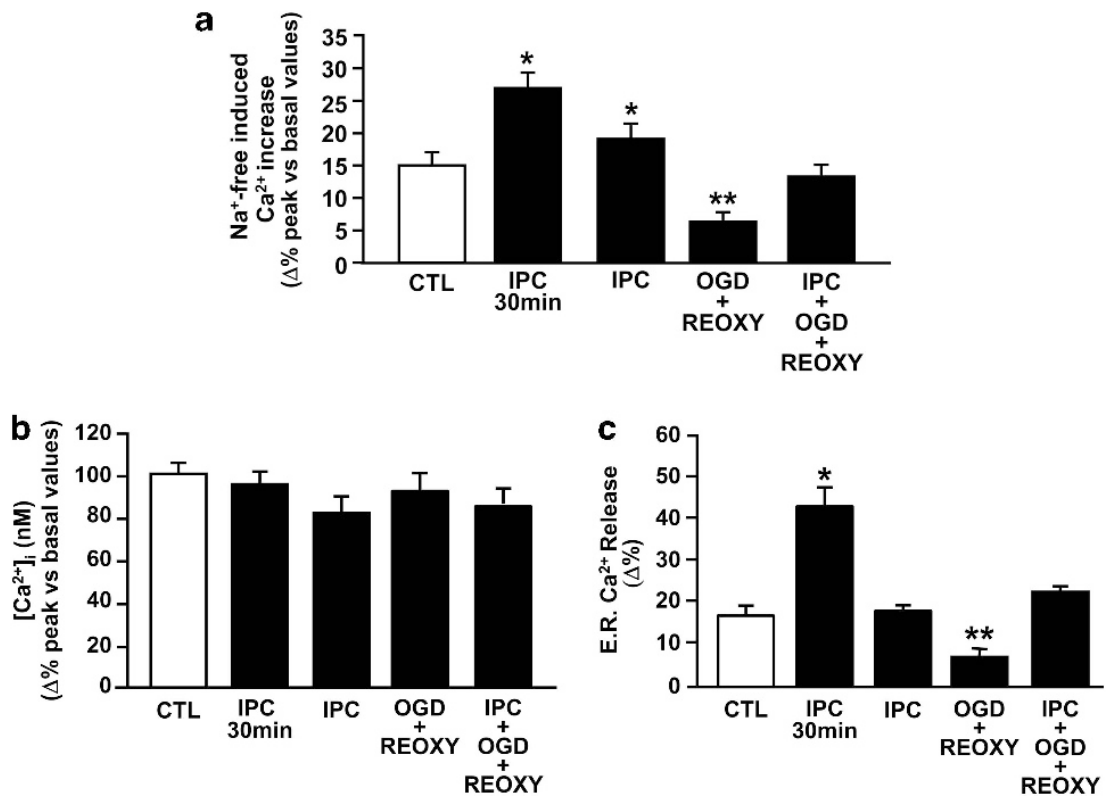

Figure $3 \mathrm{NCX}$ activity and $\left[\mathrm{Ca}^{2+}\right]_{\mathrm{i}}$ in cortical neurons exposed to IPC and IPC followed by OGD/reoxygenation. Cortical neurons (12 DIV) were exposed to IPC (30-min OGD) followed by $48 \mathrm{~h}$ of normoxia, to OGD/reoxygenation (OGD/Reoxy), or to IPC followed by OGD/Reoxy (IPC/OGD/Reoxy). Calcium measurements were performed at the end of treatments by Fura2-AM. (a) Quantification of the effect of the aforementioned conditions on $\left[\mathrm{Ca}^{2+}\right]_{\mathrm{i}}$ increase induced by the $\mathrm{Na}^{+}$-free. The percentage of $\left[\mathrm{Ca}^{2+}\right]_{\mathrm{i}}$ increase after $\mathrm{Na}^{+}$-free perfusion was calculated as the percentage change of plateau/basal value. (b) Cytosolic calcium measurements $\left[\mathrm{Ca}{ }^{2+}\right]$ i. (c) $\mathrm{ER}$ calcium concentration measured as $\left[\mathrm{Ca}^{2+}\right]_{i}$ increase after rapid perfusion of ATP $(100 \mu \mathrm{M})$ plus thapsigargin $(\mathrm{Tg}, 1 \mu \mathrm{M})$. Each bar represents the mean ( \pm S.E.M.) of the values obtained from three independent experimental sessions. ${ }^{\star} P<0.05$ versus $C T L ;{ }^{* *} P<0.05$ versus IPC, IPC $+O G D+R E O X Y$, and $C T L$ 
cytosolic calcium concentration $\left(\left[\mathrm{Ca}^{2+}\right]_{i} ;\right.$ Figure $\left.3 b\right)$, whereas it significantly increased ER calcium concentration (Figure 3c). Indeed, when cortical neurons were exposed to 30 min of IPC and then challenged with a solution containing $\mathrm{ATP}+\mathrm{Tg}$ to induce a massive calcium release from intracellular IP3-sensitive calcium stores, ${ }^{16}$ the ER calcium concentration was higher in cortical neurons exposed to IPC than in neurons exposed to OGD/reoxygenation (Figure 3c) or sham treatment.

The increased activity of NCX during IPC was strictly related to $\mathrm{NCX} 1$ and $\mathrm{NCX} 3$, as it was almost completely prevented when cells were treated with siRNA against NCX1 and NCX3, respectively (Figure 4a). However, only siNCX1 was able to prevent $\mathrm{ER} \mathrm{Ca}^{2+}$ refilling during IPC (Figure $4 \mathrm{~b}$ ). In fact, in neurons exposed to IPC, ATP + Tg-induced $\left[\mathrm{Ca}^{2+}\right]_{i}$ rise was lowered by siNCX1 but not by siNCX3 treatment (data not shown). Interestingly, the same effect was observed after neurons were pretreated with L-NAME, thus suggesting that NCX1 activation during IPC stimulates ER calcium refilling in an $\mathrm{NO}$-dependent manner.

IPC-induced NCX3 upregulation promotes mitochondrial calcium extrusion and improves mitochondrial activity. When neurons were exposed to IPC or to IPC followed by OGD/reoxygenation, $\left[\mathrm{Ca}^{2+}\right]_{\mathrm{m}}$ was reduced. This effect was prevented after siRNA-NCX3 treatment (Figure 5a). These results, obtained by X-Rhod1 measurements with confocal microscopy, were further confirmed by single cell microfluorimetry. In fact, the knocking down of NCX3 in neurons counteracted the reduction of FCCP-induced $\left[\mathrm{Ca}^{2+}\right]_{i}$ increase occurring during IPC and IPC followed by $\mathrm{OGD} /$ reoxygenation. Furthermore, the treatment of neurons with CGP37157, a selective blocker of mitochondrial NCX, ${ }^{17}$ increased mitochondrial calcium concentrations (Figure 5b).
Interestingly, when neurons were treated with siRNA against NCX1 (Figure 5c) no changes were observed.

To understand whether the reduction in mitochondrial calcium concentrations were responsible for IPC-induced neuroprotection, mitochondrial oxidative capacity was assessed in cortical neurons pretreated with CGP37157 and, subsequently, exposed to IPC. As reported in Figure $6 \mathrm{a}$, mitochondrial redox activity worsened in neurons either pretreated with CGP37157 or exposed to IPC but was unaffected by IPC in the absence of CGP37157. Moreover, the significant improvement in mitochondrial activity observed in preconditioned neurons exposed to OGD/reoxygenation was abolished when cells were treated with CGP37157 (Figure 6a). To rule out the possibility that the effect of CGP37157 was related to a modification of the mitochondrial membrane potential, it was measured by using TMRE fluorescent probe in CGP-treated neurons exposed to IPC and IPC followed by OGD/rexoygenation. The results showed that CGP37157 did not affect mitochondrial membrane potential (Figure 6b).

To further support the hypothesis that NCX1 and NCX3 were responsible for IPC-induced neuroprotection, neuronal viability was evaluated after the silencing of the two $\mathrm{Na}^{+} / \mathrm{Ca}^{2+}$ exchanger isoforms during IPC. siRNA against NCX1 and NCX3 prevented the neuroprotective effect of IPC in cortical neurons exposed to a subsequent OGD/ reoxygenation (Figure $6 c$ ).

\section{Discussion}

The results of the present study clearly demonstrated that in cortical neurons the neuroprotection elicited by preconditioning through the pathway NO/PI3K/Akt on NCX1-NCX3 activation promotes ER refilling and mitochondrial calcium a
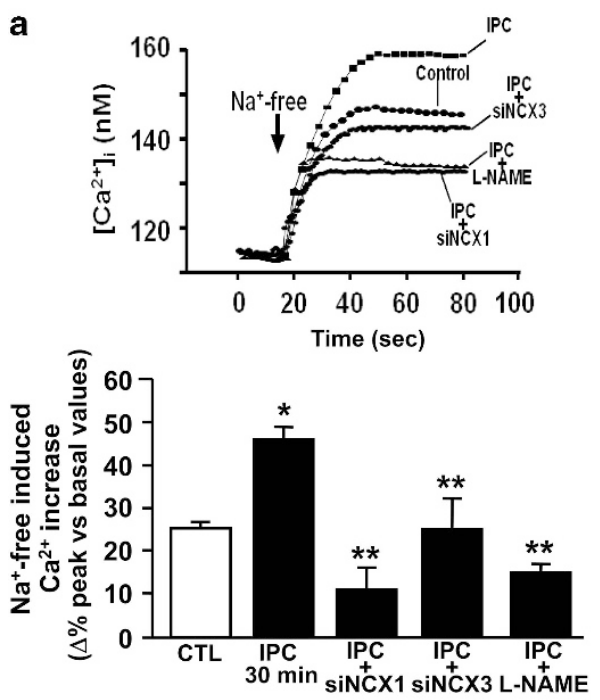

b
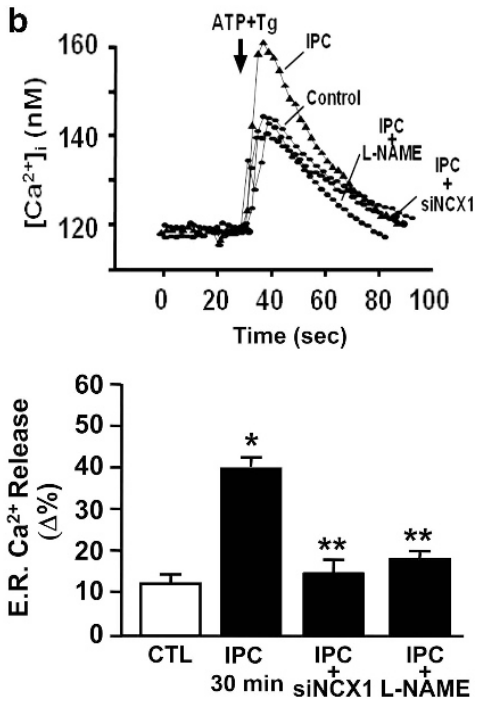

Figure 4 IPC-induced NCX activity and ER refilling in cortical neurons treated with siNCX1, siNCX3 and L-NAME. (a) Top superimposed single-cell traces representative of the effect of $\mathrm{Na}^{+}$-free on $\left[\mathrm{Ca}^{2+}\right]_{\mathrm{i}}$ in normoxic neurons, IPC neurons (30-min OGD) and IPC neurons (30-min OGD) previously treated with siNCX1, siNCX3 and L-NAME. Bottom quantification of the effect of the aforementioned conditions on $\left[\mathrm{Ca}^{2+}\right]_{i}$ increase induced by the Na ${ }^{+}$-free. (b) Top superimposed single-cell traces representative of the effect of ATP $+\mathrm{Tg}$ on $\left[\mathrm{Ca}^{2+}\right]_{\mathrm{i}}$ in normoxic neurons, IPC neurons (30-min OGD) and IPC neurons (30-min OGD) previously treated with siNCX1, and L-NAME. Each bar represents the mean ( \pm S.E.M.) of the values obtained from three independent experimental sessions. ${ }^{*} P<0.05$ versus $C T L$; ${ }^{* *} P<0.05$ versus IPC 
a
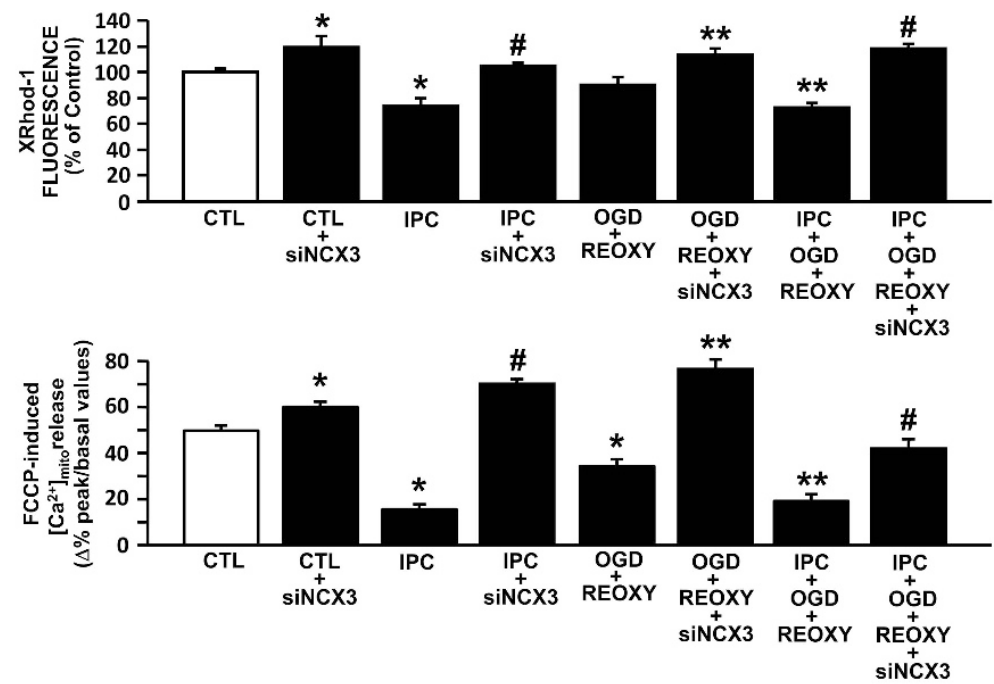

b
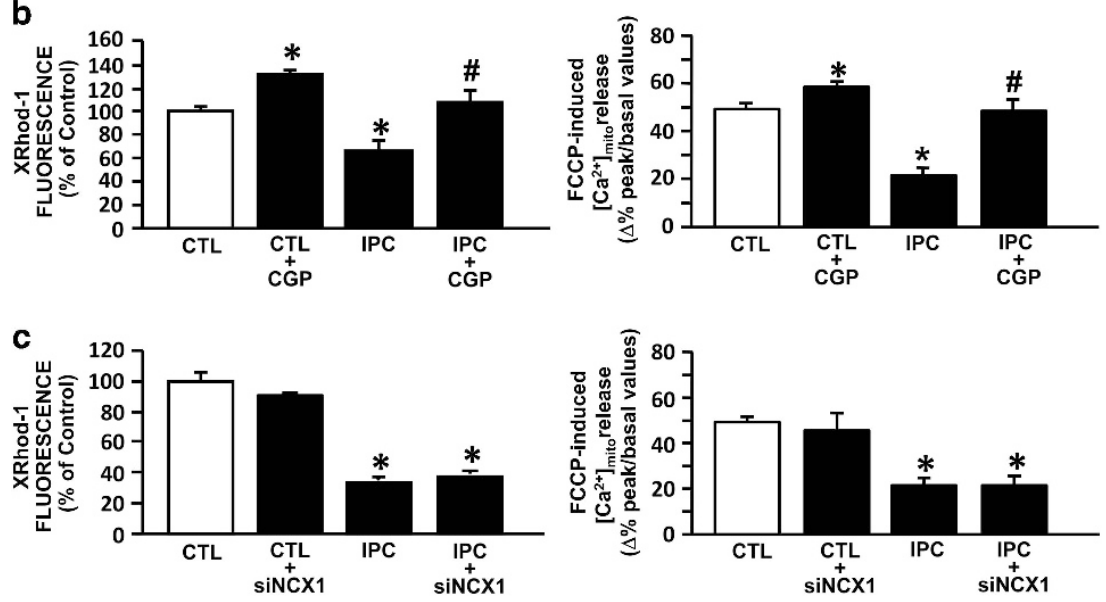

Figure $5\left[\mathrm{Ca}^{2+}\right]_{\mathrm{m}}$ in cortical neurons exposed to IPC and IPC followed by OGD/reoxygenation. (a) $\left[\mathrm{Ca}^{2+}\right]_{\mathrm{m}}$ evaluated in neurons (12 DIV) exposed to IPC (30-min OGD) followed by $48 \mathrm{~h}$ of normoxia and to $3 \mathrm{~h}$ of OGD plus $24 \mathrm{~h}$ of reoxygenation in the presence and absence of siNCX3. (b) $\left[\mathrm{Ca}^{2+}\right]_{\mathrm{m}}$ measured in neurons (12 DIV) exposed to IPC followed by $48 \mathrm{~h}$ of normoxia the presence of CGP37157. (c) $\left[\mathrm{Ca}^{2+}\right]_{\mathrm{m}}$ measured in neurons (12 DIV) exposed to IPC followed by $48 \mathrm{~h}$ of normoxia after siRNA treatment against NCX1. $\left[\mathrm{Ca}^{2+}\right]_{\mathrm{m}}$ was measured at the end of the normoxia with confocal microscopy, with the fluorescent probe X-Rhod1, and with single cell microfluorimetry, with Fura-2AM after FCCP treatment. ${ }^{*} P<0.05$ versus $C T L ;{ }^{*} P<0.05$ versus 0 GD/Reoxy; ${ }^{\#} P<0.05$ versus its respective untreated $C T L$

extrusion, thus preventing intracellular calcium dysregulation induced by OGD. Interestingly, it was demonstrated that upon the preconditioning stimulus $\mathrm{NO}$ is the messenger that triggers the activation of the PI3K/Akt pathway that in turn determines the increased expression and activity of the two isoforms, NCX1 and NCX3, of the exchanger. In particular, IPC through NO production was able to stimulate NCX activity in the reverse mode of operation. The specificity of this effect was demonstrated by its reversal obtained with siRNA against NCX1 and NCX3 and through the inhibition of NO production with L-NAME and 7NI. In the early phase of the IPC the increased activity of NCX1 in the calcium-entering mode of operation (reverse mode) favours an increased refilling of $\mathrm{Ca}^{2+}$ into the ER. This effect was exclusively due to NO-dependent NCX1 activation, since it was counteracted by siNCX1 and L-NAME treatments. This finding is in accordance with data recently published by Secondo et al., ${ }^{18}$ showing that an increase in NCX1 activity is promoted by NO. Moreover, NCX1-increased activity promotes ER
$\mathrm{Ca}^{2+}$ refilling, thereby avoiding ER stress in neurons exposed to OGD. ${ }^{6}$

Contrariwise, NO produced a raise in NCX3 expression through Akt activation in the late phase of IPC (48h). This increased expression of NCX3 does not affect cytosolic calcium concentration but is associated to a reduction of mitochondrial calcium content that was prevented by siNCX3 and CGP37157, the selective inhibitor of mitochondrial NCX. We recently demonstrated that NCX3, besides being localized on the neuronal plasmamembrane, is also expressed on the outer mitochondrial membrane, where it contributes to the extrusion of calcium from the mitochondria under physiological and pathological conditions such as hypoxia. ${ }^{9}$ Therefore, it is possible to hypothesize that the increased expression in NCX3, within $48 \mathrm{~h}$ of IPC, might exert neuroprotective effects reducing mitochondrial calcium concentration, thus improving mitochondrial oxidative capacity.

It is well known that mitochondria, in addition to generating cellular energy, have an important role in regulating 

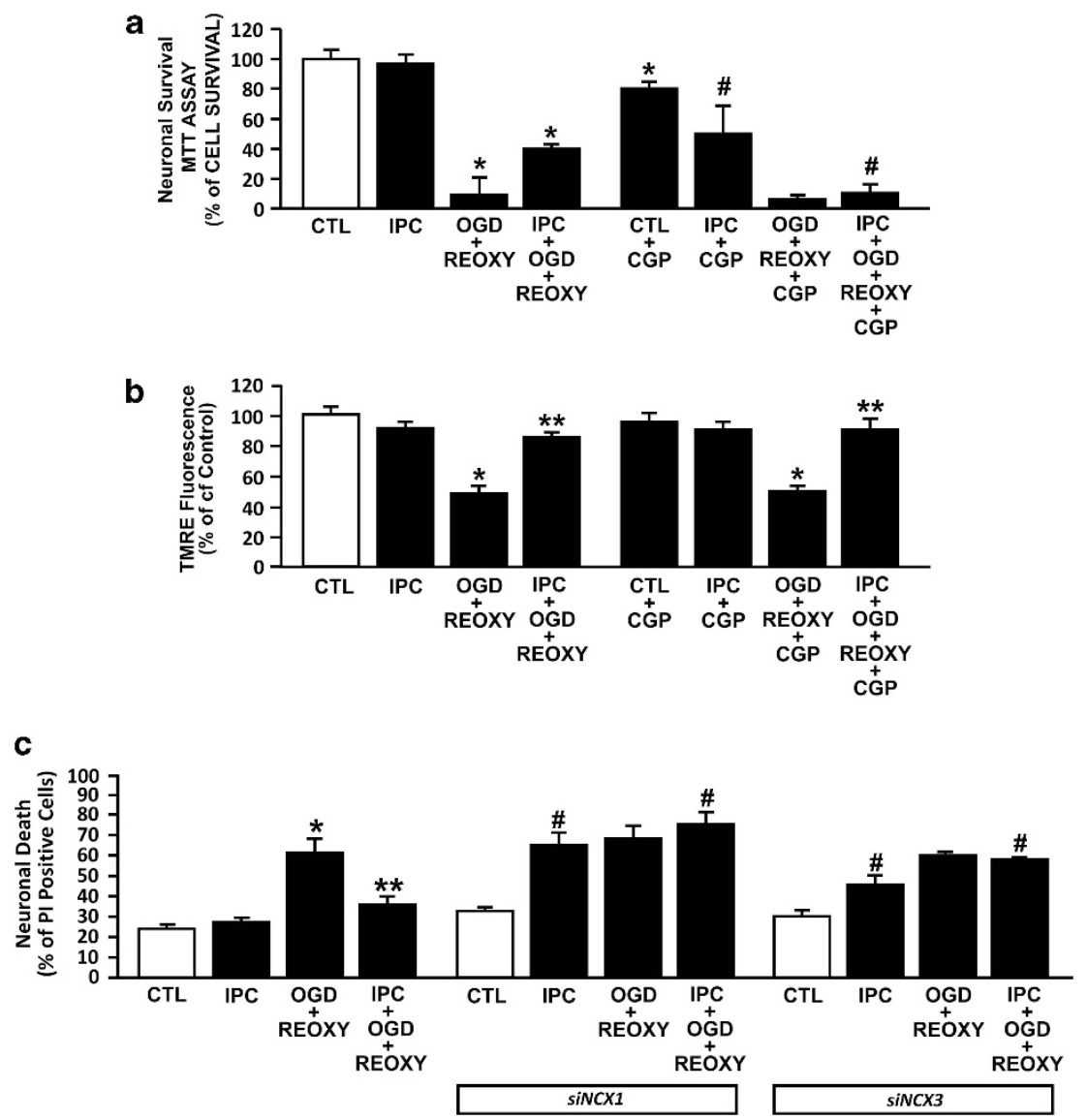

Figure 6 Mitochondrial activity and neuronal viability in cortical neurons exposed to IPC followed by OGD/reoxygenation. (a) Mitochondrial function measured by MTT in cortical neurons exposed to IPC and IPC followed by OGD/reoxygenation in the presence of CGP37157. ${ }^{*} P<0.05$ versus CTL and IPC; ${ }^{\#} P<0.05$ versus IPC and IPC + OGD/Reoxy. (b) Mitochondrial membrane potential measured by confocal microscopy in cortical neurons exposed to IPC and IPC followed by OGD/reoxygenation in the presence of CGP3715. ${ }^{\star} P<0.05$ versus $\mathrm{CTL}$ and IPC; ${ }^{*} P<0.05$ versus its respective OGD/Reoxy. (c) Cell viability measured by PI and FDA in cortical neurons exposed to IPC and IPC followed by OGD/reoxygenation in the presence of siNCX1 and siNCX3. ${ }^{*} P<0.05$ versus $C T L ;{ }^{* \star} P<0.05$ versus 0 GD/Reoxy; ${ }^{\sharp} P<0.05$ versus its respective CTL

cellular calcium homeostasis ${ }^{19-22}$ in concert with the sarco-endoplasmic reticulum $\mathrm{Ca}^{2+}$-ATPase, the plasma membrane $\mathrm{Ca}^{2+}$-ATPase and the $\mathrm{Na}^{+} / \mathrm{Ca}^{2+}$ exchanger. ${ }^{23}$ On the other hand, maintaining mitochondrial calcium homeostasis is crucial for mitochondrial function. For instance, under conditions of high cellular demand, $\mathrm{Ca}^{2+}$-sensitive dehydrogenases can regulate oxidative phosphorylation and ATP synthesis. ${ }^{24}$ Therefore, the increased expression of NCX3 observed in the late phase of IPC could be related to an improvement of the exchanger activity on mitochondria during IPC, which promotes the extrusion of calcium from the organelle. This effect renders mitochondria more resistant to the subsequent lethal OGD/reoxygenation insult. Finally, evidence that siNCX3 and CGP37157 treatments counteracted the neuroprotective effect exerted by IPC on mitochondrial oxidative capacity and cell survival further supports this hypothesis. Considering the tight correlation between mitochondrial calcium content, oxidative metabolism and neuronal survival, our present results add a new insight into the molecular mechanisms involved in IPC-induced neuroprotection. Indeed, the IPC-induced upregulation of NCX3 by helping mitochondria to preserve their energetic capacity makes them less vulnerable to the subsequent lethal insult. On the other hand, the NO-induced upregulation of NCX1 occurring in the early phase of IPC prevents ER stress, thus further contributing to IPC-induced neuronal survival. This is the first evidence showing that neuroprotection induced by IPC depends on the regulation of ER and mitochondria calcium homeostasis.

Evidence that the upregulation of NCX1 and NCX3 expression in neurons exposed to IPC depends on PI3K/Akt activation is in accordance with in vivo studies. ${ }^{1}$ Indeed, when rats subjected to middle cerebral artery occlusion (MCAO) were intracerebroventricularly (ICV) treated with the PI3K inhibitor LY294002, ${ }^{12}$ the expression of NCX1 and NCX3 was downregulated. Interestingly, the novel aspect of the present study was that IPC through the mediation of NO, PI3K/Akt and NCX1 and NCX3 interferes with ER refilling and mitochondrial calcium handling. Indeed, L-NAME and 7NI, two well-known inhibitors of NO synthesis, ${ }^{14,15}$ completely counteracted Akt activation during IPC. Moreover, L-NAME and 7NI treatments also inhibited the increase in the expression of NCX3 and in the activity of NCX1, thus suggesting that the NO/PI3K/Akt pathway activated by IPC may regulate $\mathrm{NCX} 1$ and $\mathrm{NCX} 3$ protein expression and activity. 
Collectively, we demonstrated for the first time that NCX1 and NCX3 have a key role in IPC-induced neuroprotection owing to their ability to control ER and mitochondrial calcium homeostasis through the activation of the NO/PI3K/Akt pathway.

\section{Materials and Methods \\ Primary cortical neuron preparation}

Post-natal neurons: Mixed cultures of cortical neurons from Wistar rat pups, 2-4 days old, were prepared by modifying a previously described method. ${ }^{25}$ The tissue was minced, trypsinized $\left(0.1 \%\right.$ for $15 \mathrm{~min}$ at $\left.37^{\circ} \mathrm{C}\right)$, triturated and plated on poly-D-lysine-coated coverslips. Finally, it was cultured in Neurobasal medium (Invitrogen, Life Technologies, Milan, Italy) supplemented with B-27 (Invitrogen) and $2 \mathrm{mM} \mathrm{L-glutamine.} \mathrm{Cells} \mathrm{were} \mathrm{plated} \mathrm{at} 1.8 \times 10^{6}$ on $25-\mathrm{mm}$ glass coverslips pre-coated with poly-D-lysine $(10 \mu \mathrm{g} / \mathrm{ml})$. Cultures were kept at $37^{\circ} \mathrm{C}$ in a humidified atmosphere of $5 \% \mathrm{CO}_{2}$ and $95 \%$ air and fed once a week, for a minimum of 10 days before use.

Embryonic neurons: Cortical pure neurons were prepared from brains of 16-day-old Wistar rat embryos. ${ }^{26}$ Briefly, the rats were first anesthetized and then decapitated to minimize the animals' pain and distress. Dissection and dissociation were performed in $\mathrm{Ca}^{2+} / \mathrm{Mg}^{2+}$-free phosphate-buffered saline (PBS) containing glucose $(30 \mathrm{mM})$. Tissues were incubated with papain for $10 \mathrm{~min}$ at $37^{\circ} \mathrm{C}$ and dissociated by trituration in EBSS containing DNAse, bovine serum albumine (BSA) and ovomucoid. Cells were plated at $0.8-1 \times 10^{6}$ in 12 plastic multiwells pre-coated with poly-D-lysine $(20 \mu \mathrm{g} / \mathrm{ml})$ in MEM/F12 (Life Technologies) containing glucose, $5 \%$ deactivated fetal calf serum (FCS) and $5 \%$ Horse Serum (HS, Life Technologies), glutamine and antibiotics - or at $15 \times 10^{6}$ in $100-\mathrm{mm}$ plastic Petri dishes Ara-C $(10 \mu \mathrm{M})$ was added within $48 \mathrm{~h}$ of plating to prevent non-neuronal cell growth. Neurons were cultured at $37^{\circ} \mathrm{C}$ in a humidified $5 \% \mathrm{CO}_{2}$ atmosphere and used after 12 days of culture.

All the experiments on primary cortical neurons were performed according to the procedures described in experimental protocols approved by the Ethical Committee of the 'Federico II' University of Naples.

Combined OGD. Preconditioning insult was reproduced in vitro by exposing cells to 30 min of OGD, performed in a medium previously saturated with $95 \% \mathrm{~N}_{2}$ and $5 \% \mathrm{CO}_{2}$ for $20 \mathrm{~min}$ and containing $\mathrm{NaCl} 116 \mathrm{mM}, \mathrm{KCl} 5.4 \mathrm{mM}, \mathrm{MgSO}_{4}$ $0.8 \mathrm{mM}, \mathrm{NaHCO}_{3} 26.2 \mathrm{mM}, \mathrm{NaH}_{2} \mathrm{PO}_{4} 1 \mathrm{mM}, \mathrm{CaCl}_{2} 1.8 \mathrm{mM}$, glycine $0.01 \mathrm{mM}$ and $0.001 \mathrm{w} / \mathrm{v}$ phenol red. ${ }^{8}$ Hypoxic conditions were maintained using a hypoxia chamber (temperature $37{ }^{\circ} \mathrm{C}$, atmosphere $5 \% \mathrm{CO}_{2}$ and $95 \% \mathrm{~N}_{2}$ ). At the end of incubation, the medium was replaced with another one containing normal levels of $\mathrm{O}_{2}$ and glucose. Hence, the cells were kept for $24 \mathrm{~h}$ under normoxic conditions. After $24 \mathrm{~h}$, they were exposed to $3 \mathrm{~h}$ of OGD, as previously described. To interrupt OGD, they were then removed from the hypoxic medium and placed into the normal culture medium, previously saturated with a mixture of $95 \% \mathrm{O}_{2}$ and $5 \%$ $\mathrm{CO}_{2}$ for $10 \mathrm{~min}$. Thus, reoxygenation was achieved by returning neurons to normoxic conditions ( $5 \% \mathrm{CO}_{2}$ and $95 \%$ air) for $24 \mathrm{~h}$.

Western Blot. Protein samples $(50 \mu \mathrm{g})$ were analyzed on $8 \%$ sodium dodecyl sulfate polyacrilamide gel with $5 \%$ sodium dodecyl sulfate stacking gel (SDS-PAGE) and electrotransferred onto Hybond ECL nitrocellulose paper (Amersham, Milan, Italy). Membranes were blocked with $5 \%$ non fat dry milk in $0.1 \%$ Tween-20 (TBS-T; $2 \mathrm{mM}$ Tris- $\mathrm{HCl}, 50 \mathrm{mM} \mathrm{NaCl}, \mathrm{pH} 7.5$ ) for $2 \mathrm{~h}$ at RT and subsequently incubated overnight at $4{ }^{\circ} \mathrm{C}$ in the blocked buffer with the $1: 1000$ antibody for NCX1 (polyclonal rabbit antibody, Swant, Marly, Switzerland), and with the 1:5000 antibody for NCX3 (polyclonal rabbit antibody, Philipson's Laboratory, UCLA, Los Angeles, CA, USA). The membranes were washed with $0.1 \%$ Tween 20 and incubated with the secondary antibodies for $1 \mathrm{~h}(1: 5000$; Amersham). Immunoreactive bands were detected by ECL (Amersham). Discrimination among the distinct types of extracts was ensured by running parallel western blots with the endogenous tubulin protein. The optical density of the bands was determined by Chemi Doc Imaging System (Bio-Rad, Milan, Italy). ${ }^{6}$

Imaging of mitochondrial $\mathrm{Ca}^{2+}$ and mitochondrial membrane potential. Mitochondrial calcium concentration $\left(\left[\mathrm{Ca}^{2+}\right]_{\mathrm{m}}\right)$ was assessed using the fluorescent dye X-Rhod1. Cells were loaded with X-Rhod1 $0.2 \mu \mathrm{M}$ for $15 \mathrm{~min}$ in a medium containing $156 \mathrm{mM} \mathrm{NaCl}, 3 \mathrm{mM} \mathrm{KCl}, 2 \mathrm{mM} \mathrm{MgSO}_{4}, 1.25 \mathrm{mM} \mathrm{KH}_{2} \mathrm{PO}_{4}$, $2 \mathrm{mM} \mathrm{CaCl} 2,10 \mathrm{mM}$ glucose and $10 \mathrm{mM}$ Hepes. The $\mathrm{pH}$ value was adjusted to
7.35 with $\mathrm{NaOH}$. After incubation, cells were washed three times in the same medium. An increase in mitochondrial localized intensity of fluorescence was indicative of mitochondrial $\mathrm{Ca}^{2+}$ overload.

Mitochondrial membrane potential was assessed using the fluorescent dye tetramethyl rhodamine ethyl ester (TMRE) in the 'redistribution mode'. Cells were loaded with TMRE $(20 \mathrm{nM})$ for $30 \mathrm{~min}$ in the above described medium. At the end of the incubation period, cells were washed in the same medium containing TMRE $(20 \mathrm{nM})$ and allowed to equilibrate. A decline in mitochondria-localized intensity of fluorescence was indicative of mitochondrial membrane depolarization. ${ }^{27}$

Confocal images were obtained using a Zeiss inverted 510 confocal laser scanning microscopy (Carl Zeiss, Oberkochen, Germany) and a $\times 63$ oil immersion objective. The illumination intensity of the 543 Xenon laser, used to excite X-Rhod-1 fluorescence, was kept to a minimum of $0.5 \%$ of laser output to avoid phototoxicity.

$\left[\mathrm{Ca}^{2+}\right]_{\mathrm{i}}$ measurement. $\left[\mathrm{Ca}^{2+}\right]_{\mathrm{i}}$ was measured by single cell computerassisted videoimaging. ${ }^{28}$ Briefly, cells, grown on glass coverslips, were loaded with $10 \mu \mathrm{M}$ Fura-2 acetoxymethyl ester (Fura-2AM) for $30 \mathrm{~min}$ at room temperature in normal Krebs solution containing (in $\mathrm{mM}$ ) $5.5 \mathrm{KCl}, 160 \mathrm{NaCl}, 1.2 \mathrm{MgCl}_{2}, 1.5 \mathrm{CaCl}_{2}$, 10 glucose and 10 Hepes- $\mathrm{NaOH}, \mathrm{pH}$ 7.4. At the end of the Fura-2AM loading period, the coverslips were placed into a perfusion chamber (Medical System Co. Greenvale, NY, USA) mounted onto a Zeiss Axiovert 200 microscope (Carl Zeiss) equipped with a FLUAR $\times 40$ oil objective lens. Forty to sixty-five individual cells were selected and monitored simultaneously from each coverslip. All of the results were presented as cytosolic $\mathrm{Ca}^{2+}$ concentrations. Assuming that the KD for FURA-2 was $224 \mathrm{nM}$, the equation of Grynkiewicz was used for calibration. ${ }^{29} \mathrm{NCX}$ activity was evaluated as $\mathrm{Ca}^{2+}$ uptake through the reverse mode by switching the normal Krebs' medium to $\mathrm{Na}^{+}$deficient $\mathrm{NMDG}+$ medium $\left(\mathrm{Na}^{+}{ }_{\text {-free}}\right.$ ) containing (in $\mathrm{mM}$ ) $5.5 \mathrm{KCl}, 147 \mathrm{~N}$-methyl glucamine, $1.2 \mathrm{MgCl}_{2}, 1.5 \mathrm{CaCl}_{2}, 10$ glucose and 10 HEPES, pH 7.4. These experiments were performed in the presence of the irreversible and selective inhibitor of the sarco(endo)plasmic reticulum $\mathrm{Ca}^{2+}$ ATPase thapsigargin $(\mathrm{Tg}, 1 \mu \mathrm{M})$. ER $\mathrm{Ca}^{2+}$ content was measured by inducing $\mathrm{Ca}^{2+}$ release from the organelle with the rapid administration of ATP $+\mathrm{Tg}$ $(100 \mu \mathrm{M}, 1 \mu \mathrm{M})$, whereas mitochondrial depolarization and $\mathrm{Ca}^{2+}$ extrusion were induced by the treatment with FCCP uncoupler $(250 \mathrm{nM})$. The amount of $\mathrm{Ca}^{2+}$ extruded in the cytoplasm upon ATP + TG or FCCP exposure, measured as $\left[\mathrm{Ca}^{2+}\right]_{\mathrm{i}}$ increase, are widely considered as indexes of ER or mitochondrial $\mathrm{Ca}^{2+}$ efflux, respectively.

Determination of cell death. Neuronal death was evaluated by measuring the ratio between dead and living cells. To quantify cell death after the experimental procedures, the cells were washed with normal Krebs and double stained with $36 \mu \mathrm{M}$ fluorescein diacetate (FDA) and $7 \mu \mathrm{M}$ propidium iodide (PI) for $5 \mathrm{~min}$ at $37^{\circ} \mathrm{C}$ in a phosphate buffer solution. Stained cells were examined immediately with a standard inverse fluorescence microscope at 480 and $546 \mathrm{~nm} .^{28} \mathrm{PI}$ - and FDA-positive cells were counted in three representative highpower fields of independent cultures and cell death was determined by the ratio of the number of PI-positive cells/PI + FDA-stained-positive cells. ${ }^{30}$

Determination of mitochondrial function. Neuronal survival was assessed by using 3-(4,5-dimethylthiazol-2-yl)-2,5, diphenyltetrazolium bromide (MTT). ${ }^{26}$ The assay was based on the red-ox ability of living mitochondria to convert dissolved MTT into insoluble formazan. ${ }^{31}$ Briefly, after treatments, the medium was removed and cells were incubated in $2 \mathrm{ml}$ of MTT solution $(0.5 \mathrm{mg} /$ $\mathrm{ml}$ ) for $1 \mathrm{~h}$ in a humidified $5 \% \mathrm{CO}_{2}$ incubator at $37^{\circ} \mathrm{C}$. To stop incubation, MTT solution was removed and $1 \mathrm{ml}$ dimethyl sulfoxide was added to solubilize the formazan product. The absorbance was monitored at $540 \mathrm{~nm}$ with a Perkin-Elmer LS 55 luminescence spectrometer (Perkin-Elmer Ltd, Beaconsfield, England). The data are expressed as percentage of cell viability compared with sham-treated cultures.

Materials. All the reagents were purchased from Sigma Chemicals (Milan, Italy), unless otherwise specified. LY294002 and wortmannin were supplied by Calbiochem (Inalco, Milan, Italy). L-NAME $(500 \mu \mathrm{M}), 7 \mathrm{NI}(500 \mu \mathrm{M})$, LY294002 $(25 \mu \mathrm{M})$ and $\mathrm{WMN}(1 \mu \mathrm{M})$ were present in the medium $30 \mathrm{~min}$ before exposure to IPC and during the IPC phase.

Small interfering RNA against NCX1 and NCX3. The mammalian expression vector, pSUPER.retro.puro (OligoEngine, Seattle, WA, USA), was 
used to express siRNA against NCX1 (siRNA-NCX1; NM 019268) in cortical neurons. To prepare siRNA-NCX1, a 60-base oligonucleotide and another oligonucleotide with the complementary sequence were annealed and inserted into pSUPER.retro.puro as previously reported..$^{12} \mathrm{~A}$ mismatch sequence cloned in the same vector was used as an experimental control. To knock down NCX3, the nucleotide sequence corresponding to the first nucleotide of the start codon $(+124-142$ ) of rat NCX3 (GenBank accession no. U53420) was inserted in the mammalian expression vector pSUPER.retro.puro (OligoEngine). Seventytwo hours after plating, cortical neurons were transfected with siRNA-NCX1, siRNA-NCX3 or siRNA mismatch in OPTIMEM with lipofectamine LTX (Invitrogen). After $5 \mathrm{~h}$, it was replaced with fresh medium.

Statistical analysis. Western blot analyses were repeated three times. All the other experiments were performed in triplicate and repeated at least three times. Data were expressed as mean \pm S.E.M. values and as percentages of basal values. Statistical significance among the means was determined by ANOVA followed by the Newman-Keul's test. A $P$-value $\leq 0.05$ was considered statistically significant.

Acknowledgements. We thank Dr. Paola Merolla for the editorial revision, Mr. Carmine Capitale for technical support. This work was supported by grants: COFIN2008; Ricerca finalizzata (2009) and Ricerca-Sanitaria progetto Ordinario (2008) by Ministero della Salute; PON PON01_01602 by MIUR, all to LA.

1. Pignataro G, Boscia F, Esposito E, Sirabella R, Cuomo O, Vinciguerra A et al. NCX1 and NCX3: two new effectors of delayed preconditioning in brain ischemia. Neurobiol Dis 2012; 45: 616-623.

2. Bojarski C, Meloni BP, Moore SR, Majda BT, Knuckey NW. $\mathrm{Na}+/ \mathrm{Ca} 2+$ exchanger subtype (NCX1, NCX2, NCX3) protein expression in the rat hippocampus following $3 \mathrm{~min}$ and $8 \mathrm{~min}$ durations of global cerebral ischemia. Brain Res 2008; 1189 198-202.

3. Mohammadi E, Bigdeli MR. Effects of preconditioning with normobaric hyperoxia on $\mathrm{Na}(+) / \mathrm{Ca}(2)(+)$ exchanger in the rat brain. Neuroscience 2013; 237: 277-284

4. Annunziato L, Pignataro G, Di Renzo GF. Pharmacology of brain $\mathrm{Na}+/ \mathrm{Ca} 2+$ exchanger: from molecular biology to therapeutic perspectives. Pharmacol Rev 2004; 56 633-654.

5. Annunziato L, Pignataro G, Boscia F, Sirabella R, Formisano L, Saggese M et al. ncx1, $n c x 2$, and $n c x 3$ gene product expression and function in neuronal anoxia and brain ischemia. Ann N Y Acad Sc 2007; 1099: 413-426.

6. Sirabella R, Secondo A, Pannaccione A, Scorziello A, Valsecchi V, Adornetto A et al. Anoxia-induced NF-kappaB-dependent upregulation of NCX1 contributes to Ca2 + refilling into endoplasmic reticulum in cortical neurons. Stroke 2009; 40: 922-929.

7. Dawson VL, Dawson TM. Neuronal ischaemic preconditioning. Trends Pharmacol Sci 2000; 21: 423-424.

8. Scorziello A, Santillo M, Adornetto A, Dell'aversano C, Sirabella R, Damiano S et al. $\mathrm{NO}$-induced neuroprotection in ischemic preconditioning stimulates mitochondrial Mn-SOD activity and expression via Ras/ERK1/2 pathway. J Neurochem 2007; 103: 1472-1480.

9. Scorziello A, Savoia C, Sisalli MJ, Adornetto A. Secondo A, Boscia F, et al. NCX3 regulates mitochondrial $\mathrm{Ca} 2+$ handling through the AKAP121-anchored signaling complex and prevents hypoxia-induced neuronal death. J Cell Sci 2013; 126(Pt 24): 5566-5577.

10. Dave KR, Saul I, Busto R, Ginsberg MD, Sick TJ, Perez-Pinzon MA. Ischemic preconditioning preserves mitochondrial function after global cerebral ischemia in rat hippocampus. J Cereb Blood Flow Metab 2001; 21: 1401-1410.
11. Zhang HX, Du GH, Zhang JT. Ischemic pre-conditioning preserves brain mitochondrial functions during the middle cerebral artery occlusion in rat. Neurol Res 2003; 25: 471-476.

12. Formisano L, Saggese M, Secondo A, Sirabella R, Vito P, Valsecchi V et al. The two isoforms of the $\mathrm{Na}+/ \mathrm{Ca} 2+$ exchanger, NCX1 and NCX3, constitute novel additional targets for the prosurvival action of Akt/protein kinase B pathway. Mol Pharmacol 2008; 73: 727-737.

13. Huang PL. Nitric oxide and cerebral ischemic preconditioning. Cell Calcium 2004; 36 : 323-329.

14. Knepper BR, Kurylo DD. Effects of nitric oxide synthase inhibitor NG-nitro-L-arginine methyl ester on spatial and cued leaning. Neuroscience 1998; 83: 837-841.

15. Pignataro G, Esposito E, Sirabella R, Vinciguerra A, Cuomo O, Di Renzo G et al. nNOS and p-ERK involvement in the neuroprotection exerted by remote postconditioning in rats subjected to transient middle cerebral artery occlusion. Neurobiol Dis 2013; 54: 105-114.

16. Secondo A, Taglialatela M, Cataldi M, Giorgio G, Valore M, Di Renzo G et al. Pharmacological blockade of ERG $\mathrm{K}(+)$ channels and $\mathrm{Ca}(2+)$ influx through store-operated channels exerts opposite effects on intracellular $\mathrm{Ca}(2+)$ oscillations in pituitary GH(3) cells. Mol Pharmacol 2000; 58: 1115-1128.

17. Cox DA, Conforti L, Sperelakis N, Matlib MA. Selectivity of inhibition of $\mathrm{Na}(+)-\mathrm{Ca} 2+$ exchange of heart mitochondria by benzothiazepine CGP-37157. J Cardiovasc Pharmacol 1993; $21:$ 595-599.

18. Secondo A, Molinaro P, Pannaccione A, Esposito A, Cantile M, Lippiello P et al. Nitric oxide stimulates NCX1 and NCX2 but inhibits NCX3 isoform by three distinct molecular determinants. Mol Pharmacol 2011; 79: 558-568.

19. Babcock DF, Herrington J, Goodwin PC, Park YB, Hille B. Mitochondrial participation in the intracellular Ca2 + network. J Cell Biol 1997; 136: 833-844.

20. Budd SL, Nicholls DG. Mitochondria, calcium regulation, and acute glutamate excitotoxicity in cultured cerebellar granule cells. J Neurochem 1996; 67: 2282-2291.

21. Jouaville LS, Ichas F, Holmuhamedov EL, Camacho P, Lechleiter JD. Synchronization of calcium waves by mitochondrial substrates in Xenopus laevis oocytes. Nature 1995; 377 : 438-441.

22. Werth JL, Thayer SA. Mitochondria buffer physiological calcium loads in cultured rat dorsal root ganglion neurons. J Neurosci 1994; 14: 348-356.

23. Saris NE, Carafoli E. A historical review of cellular calcium handling, with emphasis on mitochondria. Biochem Biokhimiia 2005; 70: 187-194.

24. Denton RM, McCormack JG. On the role of the calcium transport cycle in heart and other mammalian mitochondria. FEBS Lett 1980; 119: 1-8.

25. Abramov AY, Scorziello A, Duchen MR. Three distinct mechanisms generate oxygen free radicals in neurons and contribute to cell death during anoxia and reoxygenation. $J$ Neurosci 2007; 27: 1129-1138.

26. Scorziello A, Pellegrini C, Forte L, Tortiglione A, Gioielli A, lossa S et al. Differential vulnerability of cortical and cerebellar neurons in primary culture to oxygen glucose deprivation followed by reoxygenation. J Neurosci Res 2001; 63: 20-26.

27. Livigni A, Scorziello A, Agnese S, Adornetto A, Carlucci A, Garbi C et al. Mitochondrial AKAP121 links CAMP and src signaling to oxidative metabolism. Mol Biol Cell 2006; 17: 263-271.

28. Secondo A, Staiano RI, Scorziello A, Sirabella R, Boscia F, Adornetto A et al. BHK cells transfected with $\mathrm{NCX} 3$ are more resistant to hypoxia followed by reoxygenation than those transfected with NCX1 and NCX2: Possible relationship with mitochondrial membrane potential. Cell Calcium 2007; 42: 521-535.

29. Grynkiewicz G, Poenie M, Tsien RY. A new generation of $\mathrm{Ca} 2+$ indicators with greatly improved fluorescence properties. J Biol Chem 1985; 260: 3440-3450.

30. Wei H, Leeds PR, Qian Y, Wei W, Chen R, Chuang D. beta-amyloid peptide-induced death of PC 12 cells and cerebellar granule cell neurons is inhibited by long-term lithium treatment. Eur J Pharmacol 2000; 392: 117-123.

31. Hansen MB, Nielsen SE, Berg K. Re-examination and further development of a precise and rapid dye method for measuring cell growth/cell kill. J Immunol Methods 1989; 119: 203-210. 